\title{
Strategies of upper blepharoplasty in aging patients with involutional ptosis
}

\author{
Tae-Yul Lee ${ }^{1}$, Yong Ho Shin ${ }^{2}$, Jin Gyu Lee ${ }^{3}$ \\ ${ }^{I}$ Department of Plastic Surgery, Korea University College of Medicine, Seoul; ${ }^{2}$ BIO Plastic Surgery Clinic, Seoul; ${ }^{3}$ Ably Plastic Surgery Clinic, \\ Seoul, Korea
}

In many aging individuals, dermatochalasis and involutional ptosis appear together. Therefore, for functional and aesthetic purposes, ptosis correction and upper blepharoplasty are performed together. The aim of this article is to investigate factors that should be considered in order to achieve good results when simultaneously performing involutional ptosis correction and upper blepharoplasty in aging patients. Involutional ptosis is usually corrected through aponeurosis advancement in mild cases. In moderate or severe ptosis, the Muller muscle and aponeurosis are used together to correct ptosis. Using the two muscles together has the advantages of reducing lagophthalmos and increasing the predictability of outcomes after surgery. Broadly speaking, the surgical method used for involutional ptosis varies depending on the specific case, but unlike congenital ptosis, it is often not necessary to perform overcorrection. In particular, if there are problems such as severe dry-eye symptoms or risk of lagophthalmos, undercorrection should be considered. When performing ptosis correction, the surgeon should be careful not to overdo skin excision; instead, limited excision should be performed. After ptosis surgery, the brow may descend and the double fold may look too small. However, in order to make the double eyelids look larger, the surgeon should consider making the double eyelid design high rather than excising an excessive amount of skin. In some cases, to obtain more natural double eyelids and favorable results, it may be necessary to perform a sub-brow lift or forehead lift before or after involutional ptosis surgery.

\section{Keywords Eyelids / Blepharoptosis / Blepharoplasty / Aging}

Received: July 7, 2020 • Revised: July 12, 2020 • Accepted: July 12, 2020

pISSN: 2234-6163 • elSSN: 2234-6171 • https://doi.org/10.5999/aps.2020.01361 • Arch Plast Surg 2020;47:290-296

\author{
Correspondence: Jin Gyu Lee \\ Ably Plastic Surgery Clinic, \\ 562 Gangnam-daero, Gangnam-gu, \\ Seoul 06044, Korea \\ Tel: + 82-2-552-5002 \\ Fax: + 82-2-552-5021 \\ E-mail: jgleeps@gmail.com
}

This review article was prepared by the Korean Academic Association of Oculoplastic Surgery, KAOPS.

\section{INTRODUCTION}

Various changes occur in the upper eyelid with age. The skin undergoes elastosis and thinning, resulting in dermatochalasis, or laxity [1]. Involutional (senile) ptosis, as its name implies, is ptosis that occurs as part of the aging process [2]. In many older adults, skin drooping and ptosis appear together. Therefore, for functional and aesthetic purposes, ptosis correction through skin excision and double eyelid surgery are combined.

In most cases, surgery for involutional ptosis shows relatively good results. However, in order to achieve functional and aesthetically favorable results, various factors must be considered. The purpose of this article is to investigate factors that should be considered in order to achieve good results when simultaneously performing involutional ptosis correction and upper blepharoplasty in aging patients. 


\section{AGING-INDUCED CHANGES IN THE UPPEREYELID}

Aging-induced changes in the upper eyelid manifest through various findings.

The skin undergoes elastosis and thinning, resulting in dermatochalasis, or laxity. The loss of skin thickness results in dynamic wrinkles, and changes resulting from solar exposure cause hyperpigmentation of the skin. The length and width of the orbital fissure change. The lacrimal glands can become ptotic [1].

Orbital fat atrophy is another characteristic finding of aging. This age-related loss of orbital fat gives rise to a clinical appearance of "sunken upper eyelids" or "sunken globe" [3].

The horizontal palpebral fissure decreases gradually as individuals approach their $60 \mathrm{~s}$. The vertical palpebral fissure also shows gradual decreasing starting in the 50s $[1,4]$.

Changes in the orbital bone have been documented, including osseous remodeling and resorption of the midface (primarily the maxilla) and the superomedial orbital rim. These changes lead to unwanted enophthalmos [5].

\section{ETIOLOGY OF INVOLUTIONAI PTOSIS}

Involutional ptosis is often associated with changes in the aponeurosis in older patients, with more than $90 \%$ having aponeurosis-type ptosis and $10 \%$ having ptosis from other causes. The causes of involutional ptosis are varied, and levator aponeurosis dehiscence or disinsertion has been described as a common cause (Table 1) [6].

In the authors' view, levator aponeurosis attenuation is more common than levator dehiscence or disinsertion (Fig. 1).
In ptosis cases, regardless observed that in all their ptosis cases, regardless of cause, histological examinations showed a substantial reduction or absence of striated levator muscle fibers in the anterior levator, where muscle cells are normally present $[7,8]$. Clinically and histologically in a series of patients with involutional ptosis in whom they demonstrated dehiscence of the medial limb of the Whitnall ligament, a lateral displacement of the tarsal plate, and fatty degeneration of the levator muscle in the area of the Whitnall ligament $[7,8]$. The ptosis was most severe in the medial part of the lid, resulting in a "nasal droop." Intraoperatively, greater advancement of the levator muscle was necessary to correct ptosis and the muscle was moved nasally to compensate for the "nasal droop" [7].

Senescence-related enophthalmos has a strong association with eyelid ptosis, which is the likely explanation for involutional (acquired) ptosis. When upright, in enophthalmic patients, the globe sits more caudally within the orbit, causing stretching and weakening of the Whitnall ligament, ultimately resulting in ptosis $[1,3,4]$.

Patients with aponeurotic defects have a high skin crease and a thin lid, whereas patients with a fatty levator muscle tend to have a normal skin crease and a bulky lid [7].

In general, levator function is normal in involutional ptosis. However, recent reports have presented many findings of slight-

Table 1. The causes and anatomical findings of involutional ptosis

Levator aponeurosis dehiscence or disinsertion

Levator aponeurosis attenuation

Fatty degeneration of the levator muscle

Dehiscence of the medial limb/lateral displacement of the tarsal plate-nasal droop Enophthalmos

\section{Fig. 1. Intraoperative findings in an involutional ptosis patient}

(A) Signs of fatty infiltration into the levator muscle. (B) The central part of the aponeurosis was attenuated.
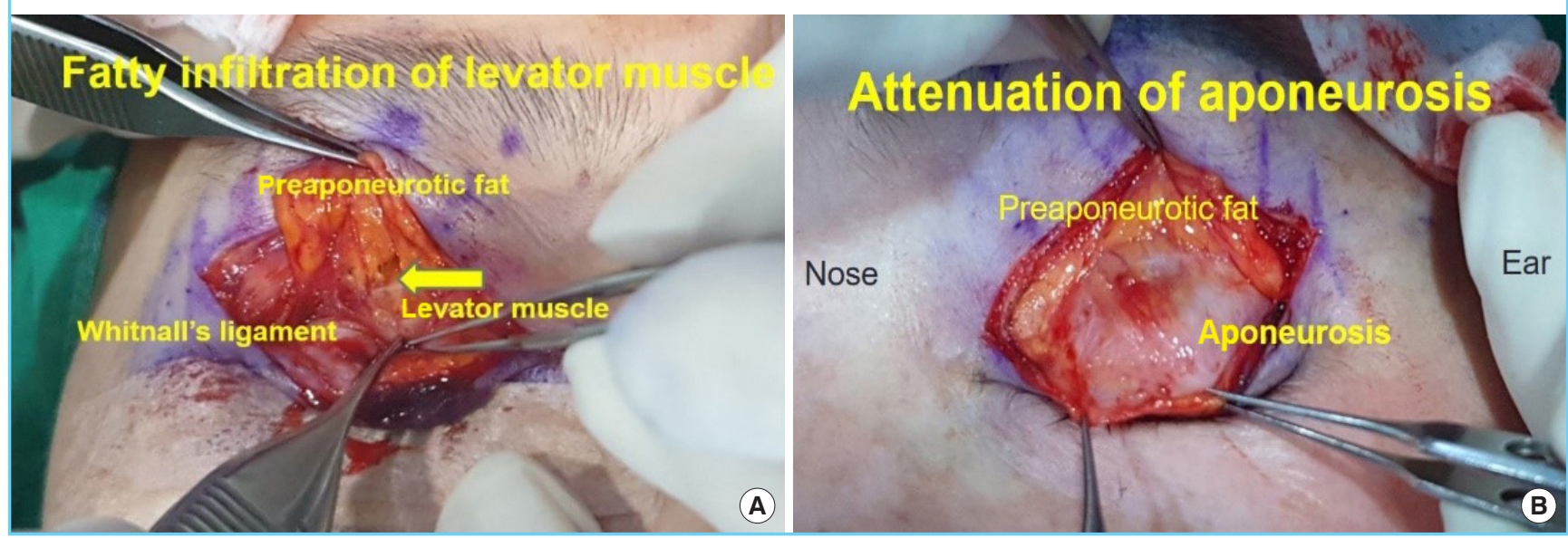
ly decreased levator function and prominent fatty infiltration in the levator muscle [9].

Involutional ptosis must be differentiated from apparent narrowing of the palpebral fissures due to the hanging down of excess skin and from herniated periorbital fat. Usual blepharoplasty suffices for both of those conditions.

A careful history should be obtained and a physical examination should be performed. The history should include the time of onset, as congenital ptosis usually involves a dysfunctional levator muscle and is treated differently from acquired ptosis [2].

\section{OPERATIVE METHODS FOR INVOLUTIONAL PTOSIS}

Various methods for correcting involutional ptosis have been reported, and can be largely divided into anterior and posterior approaches (Muller muscle conjunctiva resection, FasanellaServat) $[10,11]$. The anterior approach is used to correct excess skin on the eyelids, bulky eyelids, and skin crease problems, as well as for reoperations. Because the skin needs to be excised, most descriptions have focused on the anterior approach [7].

Methods of correcting involutional ptosis include levator reattachment, levator plication, levator advancement, and levator aponeurosis resection (Table 2).

According to Kahn [12], in cases of mild involutional ptosis due to levator weakness, levator muscle plication is recom- mended during the course of blepharoplasty. This is a simple and effective maneuver that can be easily performed in association with cosmetic blepharoplasty. However, the levator plication method is associated with a higher relapse rate than other methods.

The authors mainly use aponeurosis advancement in mild cases of involutional ptosis. In moderate or severe ptosis, the Muller muscle and the aponeurosis are used together to correct ptosis (Figs. 2, 3). The advantage of using the two muscles together is to reduce lagophthalmos and to increase predictability after surgery $[13,14]$. The advantage of using two muscles instead of just one muscle is the reduced shortening of each muscle individually. Distributing the shortening to other eye-elevating muscles and augmenting the physiological component of eyelid elevation enables the same lifting effect to be achieved with less eyelid shortening. In theory, this should induce less lagophthalmos and lid lag [15]. The levator aponeurosis and Muller muscle

\begin{tabular}{l} 
Table 2. Involutional ptosis correction methods (anterior \\
approach) [11] \\
\hline Levator reattachment \\
Levator aponeurosis plication \\
Levator aponeurosis advancement \\
Levator resection \\
Muller tuck and levator aponeurosis advancement method \\
\hline
\end{tabular}

\section{Fig. 2. Sunken eyelid and severe ptosis}

A 60-year-old male patient had no history of prior surgery on the eyelid. We designed the double eyelids to be $6 \mathrm{~mm}$ in length given the patient's sex. We performed skin excision of $3 \mathrm{~mm}$ so that the eyelid was slightly lifted when pinched. Fat repositioning was then performed to correct the sunken upper eyelid. Involutional ptosis was addressed through the Muller tuck and levator aponeurosis advancement method. Before surgery: eyes open (A), eyes closed (B). Twenty-four months after surgery: eyes open (C), eyes closed (D).
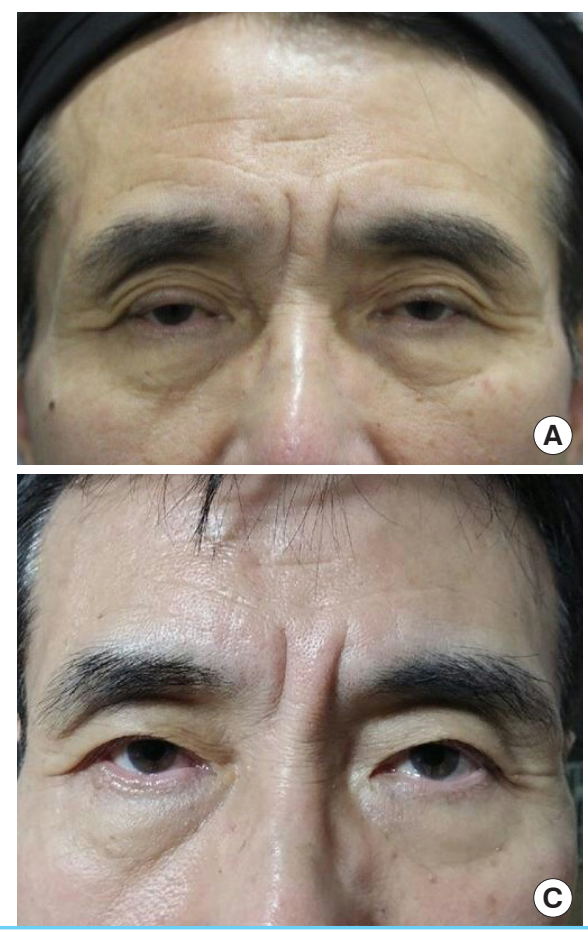
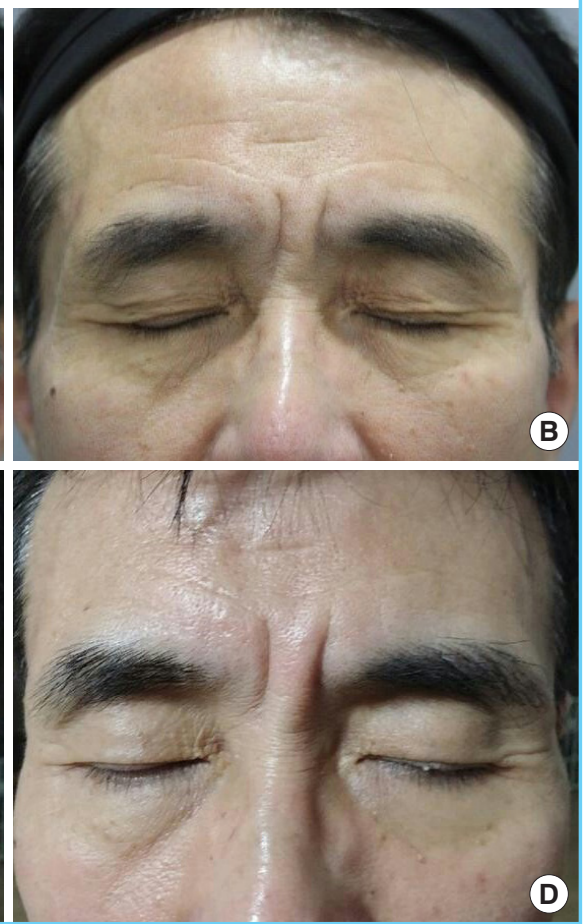


\section{Fig. 3. Right (asymmetric) ptosis}

A 58-year-old female patient opened her eyes by lifting her eyebrows. Involutional ptosis was addressed through the Muller tuck and levator aponeurosis advancement method. Lower blepharoplasty was also performed. Before surgery: eyes open (A), eyes closed (B). Fourteen months after surgery: eyes open (C), eyes closed (D).
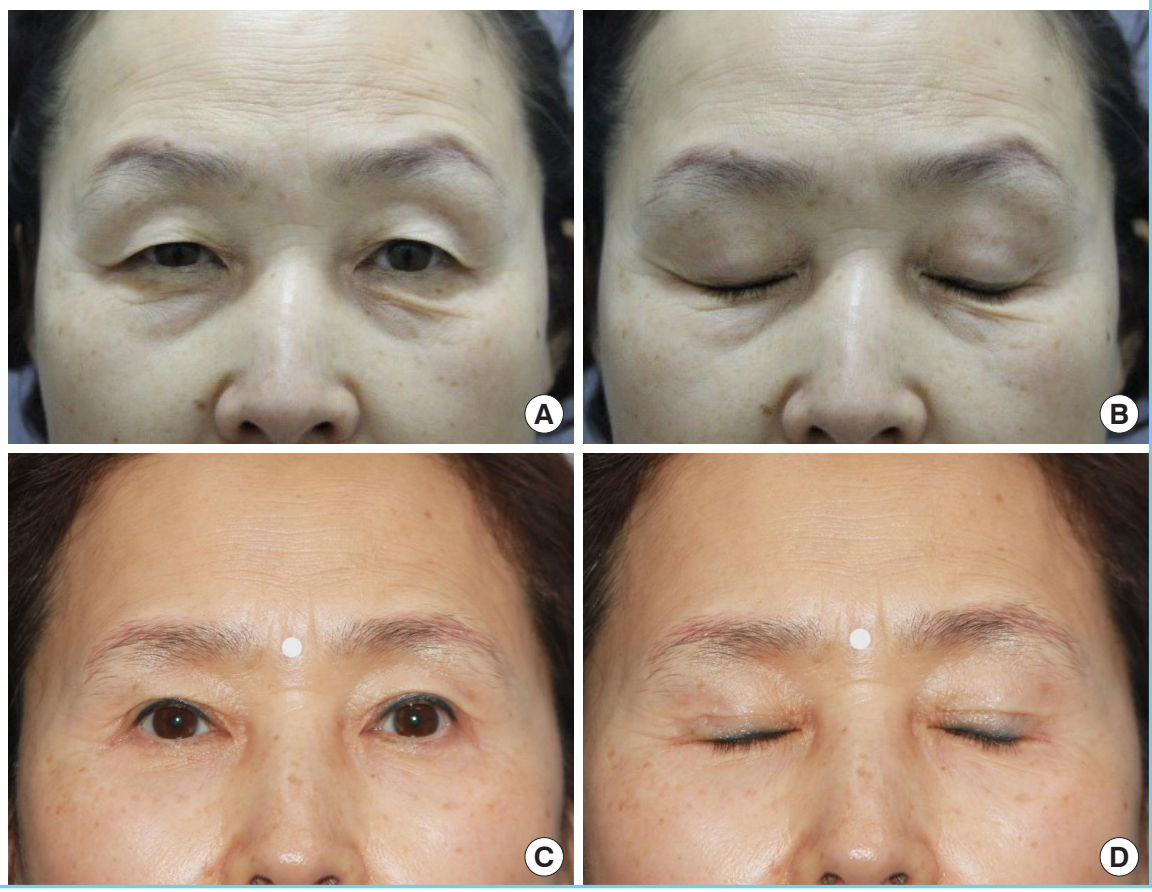

tucking procedure can reduce the amount of these structures that is resected, thereby alleviating discomfort when patients open their eyes [14].

Involutional ptosis is generally of the aponeurotic type, and therefore shows better results than other types of ptosis. In the authors' experience, even severe ptosis can be corrected using the Muller tuck and aponeurosis advancement method.

Finally, it is important to evaluate ptosis correction with the patient in the seated position during surgery.

\section{PLANNING THE AMOUNT OF PTOSIS CORRECTION}

The target for ptosis surgery is that the upper eyelid should cover the upper limbus by $1-2 \mathrm{~mm}$.

In some cases, ptosis seems to be well corrected during surgery, but after surgery, it becomes clear that it was not fully corrected due to the use of insufficient forehead muscle. In such cases, slight overcorrection is usually required.

Alternatively, in some cases, involutional ptosis appears to be overcorrected immediately after surgery, even when appropriate correction was performed. It should be explained to the patient that such cases normalize within several months, and regular follow-up should be performed.

Broadly speaking, the surgical method used for involutional ptosis varies depending on the specific case, but unlike congenital ptosis, it is often not necessary to perform overcorrection.
The authors usually use both the Muller muscle and levator aponeurosis to correct ptosis (Figs. 2, 3). Doing so is advantageous in patients who are likely to develop lagophthalmos and have a high risk of relapse. In some patients with lagophthalmos or dry-eye syndrome that is likely to worsen significantly after ptosis surgery, undercorrection of ptosis should be considered.

Elderly patients may think that the impression resulting from ptosis correction is too strong, even if the correction is within the normal range. Therefore, some practitioners consciously avoid overcorrection for certain patients, instead performing the surgery within the normal range or even performing slight undercorrection.

\section{DETERMINING THE AMOUNT OF SIKIN EXCISION}

When combined with blepharoplasty, the ellipse of skin for excision is also marked prior to surgery.

Several methods have been introduced to determine the amount of skin that should be excised during blepharoplasty in aging patients.

The pinch test, using forceps, is commonly applied to estimate the amount of excess eyelid skin to be removed. The amount of excised skin varies with the degree of dermatochalasis, and creation of 1 to $2 \mathrm{~mm}$ of lagophthalmos is the end point. Placing the inferior incision at the supratarsal crease and utilizing the pinch test while preserving at least $1 \mathrm{~cm}$ of supratarsal infrabrow 
skin safely maximizes skin excision and results in a youthful-appearing upper eyelid $[16,17]$.

Maegawa et al. [18] measured resting lid height (RLH) and maximum lid height (MLH). RLH is the distance between the lower margin of the eyelid and the lower border of the eyebrow with passive closure of the eyes. MLH is measured as the distance between the lower margin of the eyelid and the lower border of the eyebrow with manual upward stretching of the eyebrow. The height of the excised skin approximated the value of MLH minus the RLH. A mild degree of temporary lagophthalmos occurred immediately after closure of the excised area in the upper lid, but resolved after reduction of postoperative edema of the eyelids.

Maegawa method is useful as a quantitative approach to skin excision. However, it does not account for the area where the eyebrows descend postoperatively and the skin of the eyelids covers the double eyelids, making the double eyelids too small.

For some patients who want more double eyelid exposure, it may be tempting to increase the amount of skin excision, considering the degree to which the eyebrows descend. However, instead of excising more skin, it is recommended to design the double eyelid 1 to $2 \mathrm{~mm}$ higher than usual. The authors generally use forceps to measure the amount of appropriate skin excision, such that lagophthalmos does not occur and the eyelash is slightly lifted when pinched with forceps.

Removing a large amount of thin skin in the pretarsal or lower preseptal area can create unnatural double eyelids. Therefore, surgeons should avoid excising large amounts of skin in this area. In Asian patients, a combination of infrabrow excision of upper thick skin and upper blepharoplasty or endoscopic brow lift and upper blepharoplasty can be chosen to achieve a more aesthetically favorable outcome [19].

Some patients with involutional ptosis have thin skin, as well as sunken eyelid and multiple fold. Such patients usually have a longer lid-to-brow distance. Therefore, surgeons may try to rectify the sunken upper eyelid through appropriate skin excision, ptosis correction, and redeployment of fat tissues. Meanwhile, patients who have relatively thick and puffy skin may look unnatural if the skin above the double eyelid is too thick. In such cases, a more natural double eyelid may be achieved by performing conservative excision of preaponeurotic fat, medial orbital fat, and retro-orbicularis fat to reduce the volume.

\section{CHANGES IN THE BROW POSITION AFTER INVOLUTIONAL PTOSIS CORRECTION}

In general, with increasing age, the body structure and parts tend to sag. In contrast, only the eyebrow is elevated with age. The causes of this elevation are the narrowing of the visual field by blepharoptosis or upper eyelid dermatochalasis, as well as long-standing contraction of the frontalis muscle [20].

The brow position after blepharoplasty is somewhat controversial. Some authors insist that the brow position remains unchanged after surgery, while others have argued that the brow position changes after surgery $[20,21]$.

In a study investigating this issue, it was found that the change in brow height was greater after ptosis correction than after blepharoplasty. The brow position was significantly affected by ptosis correction and the distance between the upper lid margin and brow was shortened after ptosis surgery. The change in the lateral brow was less than the change in medial and central brow after ptosis surgery. Absence of the frontalis muscle in the lateral brow caused relative restriction of movement in the lateral brow [20].

Depending on the patient, the eyebrows rarely descend after ptosis surgery. The eyebrows may also descend slowly 6 months to 1 year after surgery. Therefore, it is safe to avoid excessive skin excision based on the expectation that the eyebrows will descend to a considerable extent after surgery.

Dry-eye syndrome consists of symptoms related to decreased tear production or increased tear film evaporation. Elective eyelid surgery may mechanically alter the corneoscleral and conjunctival interface sufficiently to aggravate or unmask a subclinical condition. Therefore, careful preoperative evaluation and assessment of this condition is critical before performing blepharoplasty [22-24].

The typical symptoms of dry-eye syndrome include dryness, burning, and a sandy or gritty sensation that worsens as the day goes on. Other symptoms include pain, redness, and foreign body sensation. In the immediate postoperative period, several therapies can be used to protect the cornea in patients with dryeye syndrome. In minor cases, lubricating eyedrops and ointment are standard modalities. If these options are not effective, an eye patch or temporary Frost suture can be placed [24].

In most patients, dry-eye syndrome resolves without major problems. However, patients with severe dry-eye syndrome after ptosis surgery should first receive conservative treatment, followed by an operation if the condition persists. If there is a possibility of severe dry-eye symptoms after ptosis surgery, normal correction or undercorrection should be considered depending on the patient.

\section{CONCLUSIONS}

In general, the postoperative results of surgery for involutional 
ptosis and blepharoplasty in aging patients are satisfactory. Involutional ptosis is usually corrected by aponeurosis advancement in mild cases. In moderate or severe ptosis, the Muller muscle and aponeurosis are used together to correct ptosis. The advantage of using the two muscles together is to reduce lagophthalmos and to increase predictability after surgery. Broadly speaking, the surgical method used for involutional ptosis varies depending on the specific case, but unlike congenital ptosis, it is often not necessary to perform overcorrection. In particular, if there are problems such as severe dry-eye symptoms or a risk of lagophthalmos, undercorrection should be considered. When performing ptosis correction, the surgeon should be careful not to overdo skin excision; instead, limited excision should be performed. After ptosis surgery, the brow may descend and the double fold may look too small. However, in order to make the double eyelids look larger, the surgeon should consider making the double eyelid design high rather than excising an excessive amount of skin. In some cases, to obtain more natural double eyelids and favorable results, it may be necessary to perform a sub-brow lift or forehead lift before or after involutional ptosis surgery.

\section{NOTES}

\section{Conflict of interest}

No potential conflict of interest relevant to this article was reported.

\section{Ethical approval}

The study was performed in accordance with the principles of the Declaration of Helsinki. Written informed consents were obtained.

\section{Patient consent}

The patients provided written informed consent for the publication and the use of their images.

\section{ORCID}

Tae-Yul Lee https://orcid.org/0000-0002-6511-5453

Jin Gyu Lee https://orcid.org/0000-0003-3112-3700

\section{REFERENCES}

1. Guyuron B, Harvey D. Periorbital and orbital aging: senile enophthalmos as a cause of upper eyelid ptosis. Plast Reconstr Surg 2016; 138:31e-37e.

2. Millay DJ, Larrabee WF Jr. Ptosis and blepharoplasty surgery. Arch Otolaryngol Head Neck Surg 1989;115:198-201.

3. Liang L, Sheha H, Fu Y, et al. Ocular surface morbidity in eyes with senile sunken upper eyelids. Ophthalmology 2011; 118:2487-92.

4. Park DH, Choi WS, Yoon SH, et al. Anthropometry of asian eyelids by age. Plast Reconstr Surg 2008;121:1405-13.

5. Sobel RK, Tienor BJ. The coming age of enophthalmos. Curr Opin Ophthalmol 2013;24:500-5.

6. Wilkins RB, Patipa M. The recognition of acquired ptosis in patients considered for upper-eyelid blepharoplasty. Plast Reconstr Surg 1982;70:431-4.

7. Collin JR. Involutional ptosis. Aust N Z J Ophthalmol 1986; 14:109-12.

8. Lai HT, Weng SF, Chang CH, et al. Analysis of levator function and ptosis severity in involutional blepharoptosis. Ann Plast Surg 2017;78(3 Suppl 2):S58-S60.

9. Kim CY, Lee SY. Distinct features in Koreans with involutional blepharoptosis. Plast Reconstr Surg 2015;135:1693-9.

10. Patel RM, Aakalu VK, Setabutr P, et al. Efficacy of Muller's muscle and conjunctiva resection with or without tarsectomy for the treatment of severe involutional blepharoptosis. Ophthalmic Plast Reconstr Surg 2017;33:273-8.

11. Chang S, Lehrman C, Itani K, et al. A systematic review of comparison of upper eyelid involutional ptosis repair techniques: efficacy and complication rates. Plast Reconstr Surg 2012;129:149-57.

12. Kahn S. Correction of senile (atonic) blepharoptosis during cosmetic blepharoplasty. Plast Reconstr Surg 1977;60:7259.

13. Lee JG, Shin YH. Blepharoptosis correction with Muller tuck method. J Korean Soc Aesthetic Plast Surg 2006;12:128.

14. Park JW, Shin HS, Park ES, et al. Balanced tucking of the levator muscle and Müller's muscle in blepharoptosis. J Korean Soc Plast Reconstr Surg 2006;33:149-54.

15. Baik BS, Ha W, Lee JW, et al. Adjunctive techniques to traditional advancement procedures for treating severe blepharoptosis. Plast Reconstr Surg 2014;133:887-96.

16 Zimbler MS, Prendiville S, Thomas JR. The "pinch and slide" blepharoplasty: safe and predictable aesthetic results. Arch Facial Plast Surg 2004;6:348-50.

17. Halvorson EG, Husni NR, Pandya SN, et al. Optimal parameters for marking upper blepharoplasty incisions: a 10year experience. Ann Plast Surg 2006;56:569-72.

18. Maegawa J, Kobayashi S, Yabuki Y, et al. Blepharoplasty in senile blepharoptosis: preoperative measurements and design for skin excision. Aesthet Surg J 2012;32:441-6.

19. Choi Y, Kang HG, Nam YS. Three skin zones in the asian upper eyelid pertaining to the Asian blepharoplasty. J Craniofac Surg 2017;28:892-7. 
20. Kokubo K, Katori N, Hayashi K, et al. Evaluation of the eyebrow position after levator resection. J Plast Reconstr Aesthet Surg 2017;70:85-90.

21. Lee JM, Lee TE, Lee $H$, et al. Change in brow position after upper blepharoplasty or levator advancement. J Craniofac Surg 2012;23:434-6.

22. Rees TD, Jelks GW. Blepharoplasty and the dry eye syn- drome: guidelines for surgery? Plast Reconstr Surg 1981;68: 249-52.

23. Kim EC. Diagnosis and treatment of dry eye syndrome. J Korean Med Assoc 2018;61:352-64.

24. Pacella SJ, Codner MA. Minor complications after blepharoplasty: dry eyes, chemosis, granulomas, ptosis, and scleral show. Plast Reconstr Surg 2010;125:709-18. 